\title{
Real-Time Imaging of Discrete Exocytic Events Mediating Surface Delivery of AMPA Receptors
}

\author{
Guillermo A. Yudowski, ${ }^{1 \star}$ Manojkumar A. Puthenveedu, ${ }^{1 \star}$ Dmitri Leonoudakis, ${ }^{4}$ Sandip Panicker, ${ }^{2}$ Kurt S. Thorn, ${ }^{3}$ \\ Eric C. Beattie, ${ }^{4}$ and Mark von Zastrow ${ }^{1,2}$ \\ Departments of ${ }^{1}$ Psychiatry, ${ }^{2}$ Cellular and Molecular Pharmacology, and ${ }^{3}$ Biochemistry and Biophysics, University of California at San Francisco, San \\ Francisco, California 94158, and ${ }^{4}$ California Pacific Medical Center Research Institute, San Francisco, California 94107
}

We directly resolved discrete exocytic fusion events mediating insertion of AMPA-type glutamate receptors (AMPARs) to the somatodendritic surface of rat hippocampal pyramidal neurons, in slice and dissociated cultures, using protein tagging with a pH-sensitive GFP (green fluorescent protein) variant and rapid (10 frames/s) fluorescence microscopy. AMPAR-containing exocytic events occurred under basal culture conditions in both the cell body and dendrites; potentiating chemical stimuli produced an NMDA receptor-dependent increase in the frequency of individual exocytic events. The number of AMPARs inserted per exocytic event, estimated using singlemolecule analysis, was quite uniform but individual events differed significantly in kinetic properties affecting the subsequent surface distribution of receptors. "Transient" events, from which AMPARs dispersed laterally immediately after surface insertion, generated a pronounced but short-lived (dissipating within $\sim 1 \mathrm{~s})$ increase in surface AMPAR fluorescence extending locally $(2-5 \mu \mathrm{m})$ from the site of exocytosis. "Persistent" events, from which inserted AMPARs dispersed slowly (typically over 5-10 s), affected local surface receptor concentration to a much smaller degree. Both modes of exocytic insertion occurred throughout the dendritic shaft, but remarkably, neither mode of insertion was observed directly into synaptic spines. AMPARs entered spines preferentially from transient events occurring in the adjoining dendritic shaft, driven apparently by mass action and short-range lateral diffusion, and locally delivered AMPARs remained mostly in the mobile fraction. These results suggest a highly dynamic mechanism for both constitutive and activitydependent surface delivery of AMPARs, mediated by kinetically distinct exocytic modes that differ in propensity to drive lateral entry of receptors to nearby synapses.

Key words: trafficking; glutamate; imaging; synapse; plasticity; exocytosis

\section{Introduction}

Membrane trafficking processes that deliver AMPA-type glutamate receptors (AMPARs) to the postsynaptic surface are critical for normal synaptic function and plasticity (Malinow and Malenka, 2002; Bredt and Nicoll, 2003). Constitutive trafficking of AMPARs to the plasma membrane is essential for maintaining synaptic responsiveness under basal conditions (Luscher et al., 1999), and regulated increases in surface AMPAR expression contribute to plasticity phenomena such as synaptic scaling and long-term potentiation (Lledo et al., 1998; Shi et al., 1999; Beattie

Received May 30, 2007; revised Aug. 23, 2007; accepted Aug. 26, 2007.

These studies were supported by National Institutes of Health Research Grants MH067931 (E.C.B.) and DA010711 (M.v.Z.). E.C.B. received additional support from the Forbes Norris Center for Amytrophic Lateral Sclerosis Research and the California Pacific Medical Center Research Institute. M.v.Z. is a member of the University of California at San Francisco (UCSF) Center for Neurobiology and Psychiatry and received additional support from the Friends of Langley Porter Endowed Chair for Research in Schizophrenia and Depression. We thank Stephen Heinemann, Aaron Marley, Gero Miesenbock, and James Rothman for providing critical materials and Henry Bourne, Robert Edwards, Roger Nicoll, and Tim Ryan for valuable discussion. Some of the experiments described in this study were performed in the Nikon Imaging Center at UCSF.

*G.A.Y. and M.A.P. contributed equally to this work.

Correspondence should be addressed to Mark von Zastrow, Room N212, Genentech Hall, University of California at San Francisco Mission Bay Campus, 600 16th Street, San Francisco, CA 94158-2140. E-mail: mark.vonzastrow@ucsf.edu.

DOI:10.1523/JNEUROSCI.2465-07.2007

Copyright $\odot 2007$ Society for Neuroscience $\quad 0270-6474 / 07 / 2711112-10 \$ 15.00 / 0$ et al., 2002; Rumpel et al., 2005; Kopec et al., 2006; Stellwagen and Malenka, 2006). Intracellular pathways mediating trafficking of AMPARs from and to the neuronal plasma membrane have been described previously (Carroll et al., 1999; Beattie et al., 2000; Lin et al., 2000; Park et al., 2004), and complex lateral mobility of AMPARs has been observed on the neuronal surface (Groc et al., 2004; Ashby et al., 2006; Cognet et al., 2006; Sharma et al., 2006; Bats et al., 2007; Ehlers et al., 2007). Little is known, however, about exocytic insertion events that comprise the key link between intracellular and surface trafficking of AMPARs. To begin with, it is not clear whether it is even feasible to detect such exocytic events at an individual level. If so, fundamental questions include whether elemental events mediating surface AMPAR insertion are regulated, and whether such regulation is achieved by changing the frequency of discrete exocytic events or by controlling the number of AMPARs inserted per exocytic event. Moreover, given that the majority of excitatory synapses in hippocampal pyramidal neurons are located on dendritic spines (Harris and Kater, 1994; Shepherd, 1996), the questions of where exocytic events occur and how AMPARs are delivered to spines remain mostly unresolved (Malinow and Malenka, 2002).

Previous studies of surface AMPAR delivery have necessarily relied on measures of net receptor number or activity, which can be affected by multiple processes in addition to receptor exocy- 
tosis (Sheng and Lee, 2001). Indeed, to our knowledge, elemental trafficking events mediating delivery of AMPARs to the plasma membrane have so far eluded direct observation by any method. This limitation may explain why recent efforts to define the location and regulation of AMPAR surface delivery have reached profoundly different conclusions (Passafaro et al., 2001; Adesnik et al., 2005; Gerges et al., 2006). With this in mind, we asked whether it might be possible to directly visualize individual exocytic events mediating surface insertion of AMPARs. Here, we describe a fluorescence imaging approach that achieves this goal, and its application to elucidating surface delivery of glutamate receptor 1 (GluR1)-containing AMPARs expressed in hippocampal slice and dissociated culture preparations. Our results provide unambiguous information regarding both the location and regulation of surface AMPAR insertion. They also provide unprecedented insight to the lateral movement of AMPARs immediately after surface insertion, and suggest the existence of kinetically distinct exocytic modes that differ in their ability to promote local surface delivery of AMPARs to synaptic spines.

\section{Materials and Methods}

Construction of superecliptic pHluorin-GluR1. A cDNA encoding superecliptic pHluorin (SEP) [provided by Gero Miesenbock (Yale University, New Haven, CT) and James Rothman (Columbia University, New York, NY)] preceded by a cleavable signal sequence was PCR-amplified using a previously described SEP-tagged adrenergic receptor as template (Yudowski et al., 2006), fused in-frame between codons 3 and 4 of the cDNA encoding rat GluR1 (Boulter et al., 1990) (flop; accession number P19490; provided by S. Heinemann, The Salk Institute, San Diego, CA) using $5^{\prime}$ EcoRl and $3^{\prime}$ BssHII restriction sites, and inserted into the cytomegalovirus-based mammalian expression vector pRK5. Tagging GluR1 in the same position with enhanced green fluorescent protein (EGFP) does not disrupt receptor function (Shi et al., 1998), and an essentially identical tagging strategy with SEP has been shown previously to reliably report activity-dependent changes in surface receptor number (Kopec et al., 2006).

Hippocampal culture and transfection. Hippocampal slice cultures were prepared from 6- to 9-d-old rats as described previously (Stoppini et al., 1991). Biolistic transfections were performed 2-3 d after slice preparation using the Helios Gene Gun (Bio-Rad, Hercules, CA) and $1.0 \mu \mathrm{m}$ gold particles coated with plasmid DNA per the manufacturer's protocol. Slices were imaged 3-7 d after transfection with similar results. Dissociated neuronal cultures were prepared from hippocampi of embryonic day 18 (E18)/E19 rats by papain digestion followed by brief mechanical trituration. Cells were plated on poly-D-lysine (Sigma, St. Louis, MO)treated $35 \mathrm{~mm}$ glass-bottom dishes (MatTek, Ashland, MA) and maintained in Neurobasal media (Invitrogen, Carlsbad, CA) supplemented with B27, penicillin, streptomycin, and L-glutamine as described previously (Yudowski et al., 2006). Neurons were transfected after 5-7 d in vitro (DIV 5-7) using Lipofectamine 2000 (Invitrogen) according to the manufacturer's protocol.

Live imaging of hippocampal slice and dissociated cultures. Imaging was performed using a Nikon (Garden City, NY) TE-2000E inverted microscope with a $100 \times 1.49$ numerical aperture (NA) TIRF objective or a $60 \times$ 1.45 NA TIRF objective (Nikon). A $488 \mathrm{~nm}$ single-line argon ion laser (Melles Griot, Rochester, NY) was used as a light source for both widefield and total internal reflection fluorescence microscopy (TIR-FM) illumination modes, using an optical fiber to adjust the position of the laser spot focused on the back focal plane. Both slice and dissociated cultures were imaged in $125 \mathrm{~mm} \mathrm{NaCl}, 2.5 \mathrm{~mm} \mathrm{KCl}, 2 \mathrm{~mm} \mathrm{CaCl}, 10 \mathrm{~mm}$ D-glucose, $25 \mathrm{~mm}$ HEPES, pH 7.4. Chemical stimulation of exocytic events was performed by supplementing the solution with $50 \mu \mathrm{M}$ forskolin combined with $100 \mu \mathrm{M} 3$-isobutyl 1-methyl xanthine (IBMX), a phosphodiesterase inhibitor similar in pharmacological activity to rolipram used previously to potentiate synaptic transmission (Otmakhov et al., 2004), or with $200 \mu \mathrm{M}$ glycine in the absence of added $\mathrm{Mg}^{2+}$ (Lu et al., 2001). Rapid imaging of neurons in the Gly/0 Mg condition was per- formed in the presence of $0.5 \mu \mathrm{M}$ tetrodotoxin and $200 \mu \mathrm{M}$ picrotoxin (Sigma) to reduce experimental variability. In some experiments, as indicated, the NMDA receptor antagonist 2-amino-5-phosphonovaleric acid (APV) (Sigma) was added before either forskolin/IBMX or Gly/0 $\mathrm{Mg}$. Temperature was controlled at $37^{\circ} \mathrm{C}$ either using a thermoelectric stage (Bioscience Tools, San Diego, CA) and objective warmer (Bioptechs, Butler, PA) or a microscope enclosure (Nikon) maintained at $37^{\circ} \mathrm{C}$ with $5 \% \mathrm{CO}_{2}$. Images were acquired at a continuous rate of 10 frames/s using a Cascade II, QuantEM 512C (both from Photometrics, Tucson, AZ), or iXon + (Andor, Belfast, UK) EM-CCD camera operated in the linear range. For post hoc analysis, coverslips were marked to indicate the region imaged live and then quickly transferred to $-20^{\circ} \mathrm{C}$ methanol for 10 min. Fixed specimens were blocked with 3\% BSA, 2\% goat serum, and $0.2 \%$ Triton X-100 in PBS for $>1 \mathrm{~h}$. Anti-postsynaptic density-95 (PSD-95) antibodies (1:500; Affinity Bioreagents) diluted in blocking buffer were added overnight at $4^{\circ} \mathrm{C}$, washed with $\mathrm{PBS}$, and incubated with goat anti-mouse Alexa Fluor 633 for $45 \mathrm{~min}$ at room temperature. Cells were then washed with PBS and mounted with Fluoromount G (Electron Microscopy Sciences, Fort Washington, PA). Images of fixed neurons were registered with the corresponding live images coverslips using the fiduciary mark and comparison of neuronal morphology evident from SEP-AMPAR fluorescence still visible in fixed specimens. The relatively low transfection efficiency (5-10\%) typically obtained allowed unambiguous registration of individual, SEP-AMPARexpressing neurons in live and fixed images. Aligned images were merged in still frames using Adobe Photoshop and in movies using MetaMorph (Molecular Dynamics, Sunnyvale, CA).

Unless indicated otherwise, images shown represent raw data with simple background subtraction of the averaged blank field intensity. For quantification of SEP-AMPAR intensity changes over prolonged time intervals, background-subtracted intensity values were corrected for photobleaching (during the image collection) by normalizing to a static region of a neighboring dendrite imaged in the same field. To quantify the frequency of insertion events graphically, SEP-AMPARs present initially in the plasma membrane were deliberately photobleached to 20 $30 \%$ of the initial surface brightness before time-lapse imaging. After this manipulation, newly inserted (previously nonfluorescent) SEPAMPARs were readily distinguished as pronounced "spikes" of increased fluorescence intensity in maximum intensity plots generated from image series with MetaMorph analysis software (Molecular Devices). To estimate the absolute frequency of insertion events in the cell body, numbers of observed transient and persistent events were tabulated from $60 \mathrm{~s}$ TIR-FM image series, and then event frequencies were normalized to the surface area analyzed. To estimate the absolute insertion event frequency in dendrites, transient and persistent events were tabulated in $60 \mathrm{~s}$ widefield image series, and then event counts were normalized per linear micrometer of dendrite visualized. Differences in event frequencies between treatment conditions were evaluated by the Mann-Whitney test; a value of $p<0.05$ [computed using Graph Pad (San Diego, CA) Prism software] was considered significant.

Single-molecule analysis. SEP-AMPAR number was estimated using an EM-CCD camera (QuantEM; Photometrics) that is capable of linear detection over a wide (16 bit) dynamic range. Peak surface fluorescence intensity of SEP-AMPAR insertion events was estimated by TIR-FM image series using maximum values collected from sequential $100 \mathrm{~ms}$ exposures. The same laser intensity and illumination angle was then used to image natively folded EGFP purified from Escherichia coli, after adsorption to poly-D-lysine-coated coverslips at pH 7.4 in the same buffer used for imaging neurons. EGFP was chosen because calculations from published data (Patterson et al., 1997; Miesenbock et al., 1998; Sankaranarayanan et al., 2000) indicate that EGFP is closely similar in brightness to SEP when excited at $488 \mathrm{~nm}$ in neutral media, and because EGFP is established to exist mostly as a monomer in aqueous solution (Stepanenko et al., 2004). The dilution factor of purified EGFP from concentrated stock $(1 \mathrm{mg} / \mathrm{ml})$ was adjusted empirically (typically $>10,000$-fold) to yield diffraction-limited spots of adsorbed fluorescent protein that were well separated $(>2 \mu \mathrm{m})$ from one another in TIR-FM images. Single molecules (defined by characteristic blinking and singlestep photobleaching) were imaged under identical conditions as live neu- 
rons, except that $300 \mathrm{~ms}$ integration was used. We found that this longer integration time yielded higher signal-to-noise ratio for more accurate estimation of single-molecule fluorescence. Accordingly, for subsequent display and analysis relative to exocytic events, background-subtracted single-EGFP fluorescence intensities (measured at $300 \mathrm{~ms}$ integration) were divided by a factor of 3 to normalize to $100 \mathrm{~ms}$ equivalent exposure.

\section{Results}

Real-time visualization of surface AMPAR insertion in hippocampal slice culture

GluR1-containing AMPARs were selectively visualized in the plasma membrane by tagging the amino-terminal ectodomain of the rat GluR1 subunit with SEP, a variant of the green fluorescent protein that is brightly fluorescent when exposed to $\mathrm{pH} 7.4$ extracellular media and nearly nonfluorescent when exposed to the acidic environment of the endocytic pathway (Miesenbock et al., 1998; Sankaranarayanan et al., 2000). We focused on GluR1-containing AMPARs because their net expression in the neuronal plasma membrane is rapidly increased in response to plasticity-inducing stimuli (Shi et al., 1999; Hayashi et al., 2000; Passafaro et al., 2001; Kopec et al., 2006). The SEP-tagging strategy applied in the present study was used previously to indicate plasticityinduced changes in net surface AMPAR number (Kopec et al., 2006), and tagging with a closely similar green fluorescent protein variant (EGFP) was shown not to disrupt GluR1 function assessed electrophysiologically (Shi et al., 1998). SEP-GluR1 was expressed in cultured hippocampal slices using biolistic transfection. We opted to image transfected slices using wide-field illumination, because this afforded relatively high photon collection efficiency useful for rapid imaging (see below). Because of significant out-of-plane fluorescence inherent to wide-field imaging, however, epifluorescence images of neurons in hippocampal slice culture differed considerably from the appearance of "optical sections" generated using confocal or multiphoton methods. Nevertheless, we were able to easily resolve individual transfected neurons in sparsely transfected slice preparations, even at low magnification in which the contribution made by out-of-plane fluorescence was greatest (Fig. $1 A ; A$, inset). Supplementing the (magnesium-deficient) imaging medium with $200 \mu \mathrm{M}$ glycine (Gly/0 Mg), a chemical manipulation reported previously to enhance surface AMPAR expression ( $\mathrm{Lu}$ et al., 2001), increased SEP-AMPAR fluorescence by $30-40 \%$ over a period of $10 \mathrm{~min}$ (Fig. $1 B$ ). We further confirmed that forsko-
A

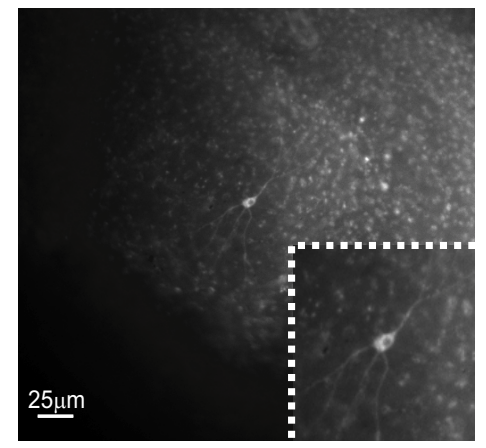

B

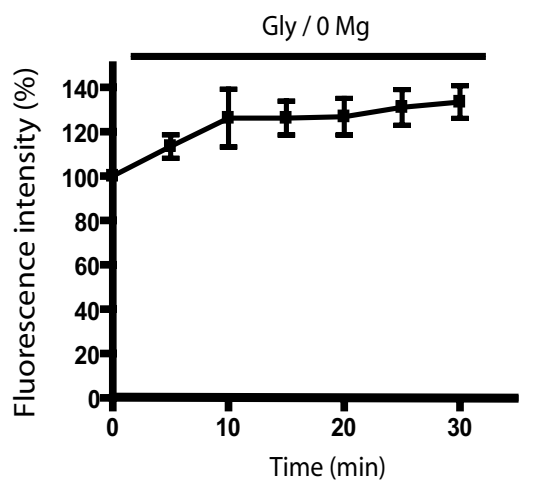

C
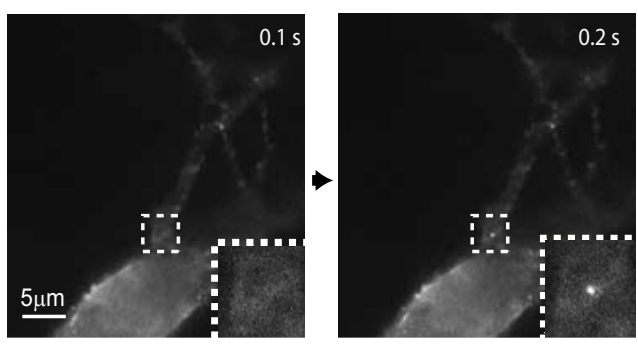

D
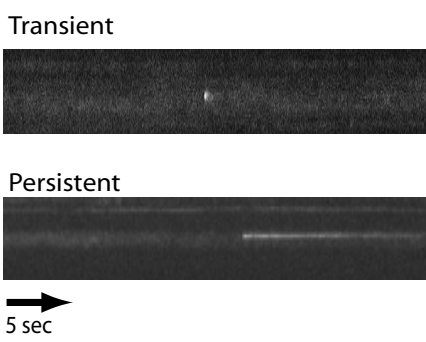

$E$

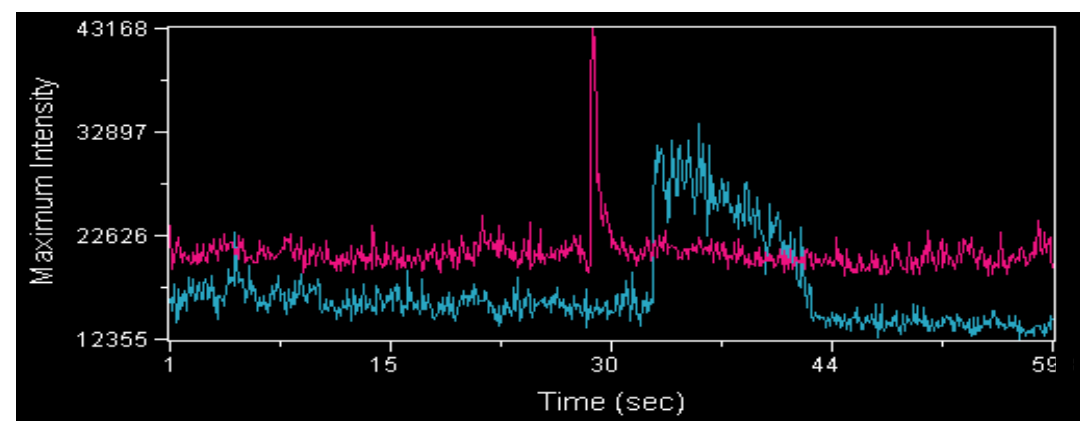

$\mathrm{F}$

G

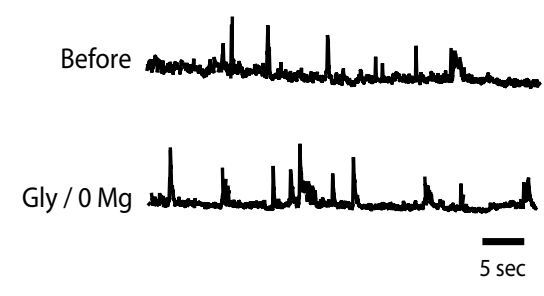

Figure 1. Detection of SEP-AMPAR puffs in cultured hippocampal slices. $\boldsymbol{A}$, Representative low magnification view of SEPAMPAR fluorescence visualized in area CA1 of a cultured hippocampal slice $5 \mathrm{~d}$ after biolistic transfection; the inset shows a transfected pyramidal neuron. $\boldsymbol{B}$, Effect of $\mathrm{Gly} / 0 \mathrm{Mg}$ on steady-state SEP-AMPAR fluorescence integrated over a single neuron. The graph shows change relative to initial value (before $\mathrm{Gly} / 0 \mathrm{Mg}$ application). Mean values were calculated across multiple experiments $(n=6)$; error bars represent SEM. C , Rapid imaging at higher magnification of SEP-AMPAR fluorescence in a field containing a neuronal cell body and proximal dendrites. Sequential $100 \mathrm{~ms}$ frames show an SEP-AMPAR insertion event (boxed area; the inset shows this region in greater detail). D, Representative kymographs illustrating the distinct kinetic properties of transient and persistent SEP-AMPAR events. $\boldsymbol{E}$, Time course of SEP-AMPAR surface fluorescence intensity of representative transient and persistent events. $\boldsymbol{F}$, Representative maximum intensity plot before (top) and immediately after (bottom) Gly/0 Mg manipulation. $\mathbf{G}$, The squares and solid line indicate cumulative frequency analysis of SEP-AMPAR events imaged during sequential 1 min intervals surrounding the indicated time points after $\mathrm{Gly} / 0 \mathrm{Mg}$ manipulation. Frequencies are normalized to $t=0$, the time interval immediately before magnesium depletion $(n=5)$. The triangles and solid line represent the same analysis conducted on cultures to which $50 \mu \mathrm{M}$ APV was added $10 \mathrm{~min}$ before $t=0(n=5)$. Error bars represent SEM. 
lin $(50 \mu \mathrm{M})$ and IBMX $(10 \mu \mathrm{M})$, another chemical manipulation essentially identical with that used previously to increase surface AMPAR expression (Otmakhov et al., 2004; Boehm et al., 2006; Kopec et al., 2006), enhanced SEP-AMPAR surface fluorescence in our preparations to a similar degree and over a similar time course (data not shown).

Because individual exocytic events are expected to occur with rapid (i.e., on the order of milliseconds) kinetics (Sankaranarayanan et al., 2000), we next worked to increase the rate of image acquisition. Using an electron-multiplying CCD detector, and collecting images in the wide field using a high numerical aperture objective, we were able to enhance the temporal resolution of our imaging studies $\sim 100$-fold (to 10 frames/s). Doing so revealed the existence of diffraction-limited "puffs" of SEPAMPAR fluorescence appearing abruptly in the preparation (Fig. $1 C)$. These events appeared within a single $(100 \mathrm{~ms})$ frame and were remarkably bright in rapid image series, typically displaying peak fluorescence intensity severalfold brighter than that observed elsewhere on the plasma membrane. Such events were observed frequently on dendrites, both close to the cell body (supplemental movie 1, available at www.jneurosci.org as supplemental material) and in distal portions (supplemental movie 2, available at www.jneurosci.org as supplemental material).

Simple visual observation revealed that individual SEPAMPAR puffs differed considerably in kinetics after their initial abrupt $(<100 \mathrm{~ms})$ appearance. Some SEP-AMPAR puffs dissipated to the level of surrounding receptor fluorescence intensity quickly, typically within several hundred milliseconds. Other puffs remained as discrete spots of SEP-AMPAR fluorescence for a more prolonged time period, typically $5-10 \mathrm{~s}$, dissipating gradually over this time period. These kinetic differences were visually obvious in kymographs (Fig. 1D) and intensity plots (Fig. 1E) generated from image series. We defined "transient" puffs as those dissipating to the level of surrounding surface fluorescence within $\leq 1 \mathrm{~s}$, and "persistent" puffs as those remaining as visible spots over the surrounding plasma membrane for $>1 \mathrm{~s}$. Using these criteria, and imaging across multiple transfected slice preparations ( $n=7$ specimens representing 209 events), persistent events represented $65.2 \pm 9.8 \%$ of the total observed in dendrites and $33.1 \pm 7.4 \%$ of the total observed on the cell body.

It was possible to recognize SEP-AMPAR puffs as abrupt peaks in maximum intensity plots generated from time-lapse image series after photobleaching of preexisting surface fluorescence (see Materials and Methods), facilitating rapid and unbiased measurement of event frequency under various conditions (Fig. 1F). Using this approach, both Gly/0 Mg (Fig. 1G) and forskolin/IBMX (data not shown) were found to increase the frequency of SEP-AMPAR puffs in a manner that was blocked by the NMDA receptor antagonist APV. Together, these results suggest that the observed puffs of abruptly increased surface SEPAMPAR fluorescence represent discrete exocytic insertion events contributing to both constitutive and regulated (NMDA receptor-dependent) components of surface AMPAR insertion.

\section{Resolving distinct exocytic insertion modes in dissociated pyramidal neurons}

To further investigate the nature of SEP-AMPAR puffs, we next asked whether similar events occur in dissociated neuronal cultures. Dissociated cultures allowed relatively detailed neuronal morphology (including dendritic spines) to be resolved in widefield epifluorescence images (Fig. 2A; $A$, inset), and facilitated the application of additional experimental approaches that are not feasible in the slice. We first used TIR-FM to examine specifically events occurring in or very near the plasma membrane (Steyer and Almers, 2001) and focused on the cell body, whose basal surface was located uniformly within the evanescent illumination field. TIR-FM clearly detected SEP-AMPAR puffs in the cell body; each event appeared as a bright spot in the maximum projection view of sequential $100 \mathrm{~ms}$ TIR-FM images (Fig. $2 \mathrm{~B}$; inset shows individual frames of the insertion event outlined by the box). Lateral spreading of SEP-AMPARs was evident in timelapse image series, particularly from transient events that produced a visually obvious increase in the surrounding plasma membrane surface (supplemental movie 3, available at www. jneurosci.org as supplemental material), suggesting that SEPAMPAR puffs represent receptor-containing exocytic events. Dendrites were incompletely illuminated by the thin $(\sim 100$ $\mathrm{nm}$ ) evanescent illumination field but were readily visualized in the wide field; therefore, rapid imaging of dendrites was generally performed using wide-field epifluorescence microscopy rather than TIR-FM. As in the slice preparation, kinetically distinct SEP-AMPAR puffs were observed in dendrites of dissociated pyramidal neurons (Fig. 2C,D). Also consistent with results obtained in transfected slice cultures, the relative frequency of persistent events observed in dissociated pyramidal neurons was significantly higher in dendrites compared with the cell body $(31.9 \pm 3.6$ compared with $10.1 \pm 4.1 \%, p=$ 0.04 ; determined from analysis of 187 events imaged in seven cells). To estimate overall event frequency, regions of randomly selected cell bodies (imaged by TIR-FM) and microscopic fields containing multiple dendrites (imaged in the wide field) were evaluated. The total number of SEP-AMPAR puffs observed (persistent and transient combined) was normalized either to the area (cell body) or length (dendrites) of each structure examined (see Materials and Methods). This analysis estimated a frequency of $0.066 \pm 0.011$ events $\mu \mathrm{m}^{-2}$ $\min ^{-1}$ in the cell body ( $n=178$ events from 10 cells) and $0.032 \pm 0.008$ events $\mu \mathrm{m}^{-1} \mathrm{~min}^{-1}$ in dendrites $(n=72$ events from eight fields). In both compartments, the observed SEPAMPAR puffs were quenched rapidly (within $\sim 1 \mathrm{~s}$ ) after addition of a membrane-impermeant acidic buffer (25 mM MES, $\mathrm{pH}$ 5.25) to the culture medium (supplemental Fig. 1, available at www.jneurosci.org as supplemental material). Together, these findings indicate that SEP-AMPAR puffs represent discrete exocytic events mediating surface insertion of GluR1-containing AMPARs in both the cell body and dendrites.

The frequency of SEP-AMPAR exocytic events was significantly increased by Gly/0 Mg (Fig. 2 E) and forskolin/IBMX (data not shown). Interestingly, the Gly/0 Mg manipulation increased the frequency of exocytic events even in the presence of TTX, consistent with previous results suggesting that the Gly/0 Mg manipulation does not require depolarization to increase the steady-state number of surface AMPARs ( $\mathrm{Lu}$ et al., 2001). This effect, and its blockade by APV, was established across multiple experiments (Fig. $2 F$ ). Whereas the frequency of SEP-AMPAR insertion events was strongly increased by chemical stimulation, the peak fluorescence intensity of individual events was not significantly changed (Fig. 2G). Furthermore, chemical stimulation did not detectably change the proportion of persistent events observed in either the cell body $(10.1 \pm 4.1 \%$ under basal conditions and $9.2 \pm 5.9 \%$ in the presence of Gly/ $0 \mathrm{Mg} ; n=350$ events) or dendrites $(31.9 \pm 3.6 \%$ under basal conditions and $35.3 \pm$ $6.1 \%$ in the presence of Gly/0 Mg; $n=270$ events). These results suggest that kinetically distinct components of surface AMPAR insertion, occurring both in the cell body and dendrites, are me- 
diated by exocytosis of a relatively uniform population of AMPAR-containing vesicles. Furthermore, they suggest that chemical stimuli that increase surface AMPAR number at steady state do so by enhancing the frequency of both transient and persistent AMPAR-containing exocytic events.

The ability to resolve discrete exocytic events mediating both constitutive and regulated surface delivery of AMPARs raised the question of how many AMPARs are inserted by each exocytic event. To address this, we determined the mean fluorescence intensity of single green fluorescent protein molecules visualized by TIR-FM (see Materials and Methods), and then compared this value to that of individual SEP-AMPAR insertion events imaged by TIR-FM of the cell body, acquired under identical conditions and in the linear detection range of the camera. We chose to calibrate single-molecule fluorescence using purified EGFP because this protein is well established to exist mostly as a monomer in solution (Stepanenko et al., 2004), and previous data indicate that EGFP is closely similar in fluorescence intensity to SEP when imaged at $\mathrm{pH} 7.4$ (Patterson et al., 1997; Miesenbock et al., 1998; Sankaranarayanan et al., 2000), the $\mathrm{pH}$ of our imaging medium. The fluorescence intensity of EGFP monomers, confirmed in our preparation by characteristic blinking and single-step photobleaching behavior (Moerner et al., 1999), resembled a Gaussian distribution (Fig. 3A,B). Individual SEP-AMPAR insertion events ranged in surface brightness from 18 to 146 times the mean measured for single molecules, with most events distributed around a single peak of surface fluorescence intensity (Fig. 3C). By calculating the mean and SD of this distribution, and normalizing to single-molecule fluorescence intensity (see Materials and Methods), we estimated that individual SEPAMPAR exocytic events deliver $51 \pm 36$ SEP-GluR1 subunits (see Materials and Methods) (Fig. 3). It was not possible to carry out a similar analysis of SEP-AMPAR fluorescence intensity on spines because of the shallow field of evanescent illumination. We therefore used wide-field epifluorescence illumination, and compared the average SEP-AMPAR fluorescence intensity measured on spines at steady state to that of individual exocytic events imaged under the same conditions and in the same specimens. This analysis revealed that, on average, the SEP-AMPAR fluorescence intensity of individual exocytic events was 2.4-fold higher than that of dendritic spines. These results suggest that the mean
A

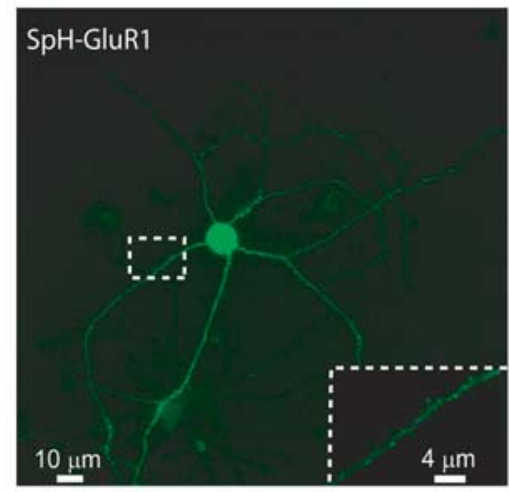

B

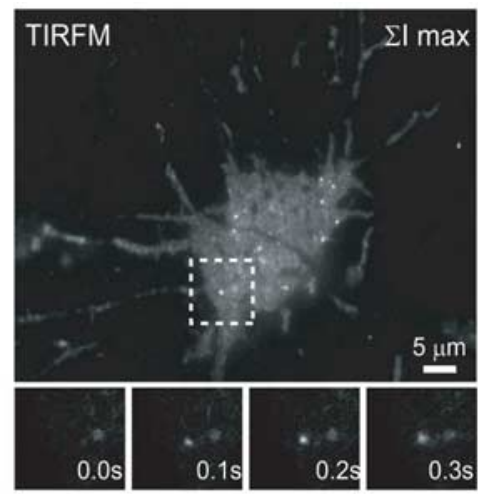

C

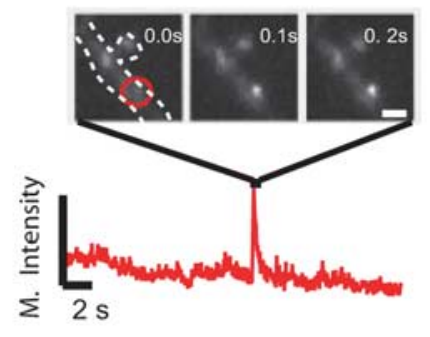

D

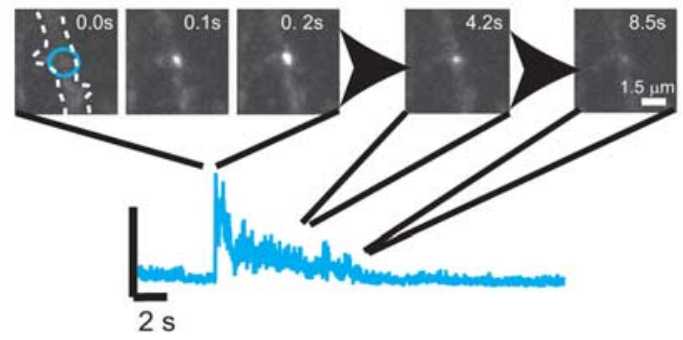

$E$

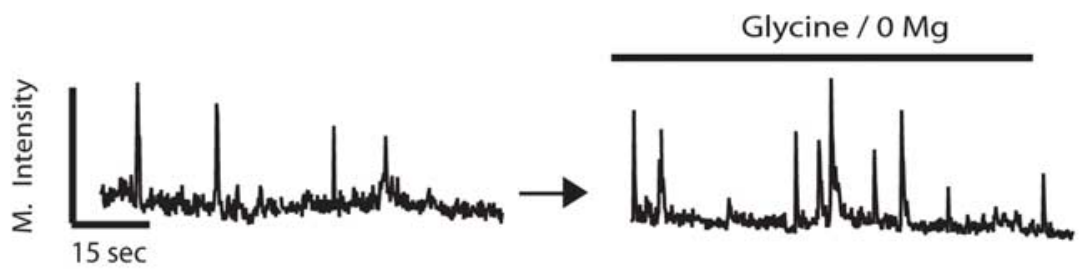

$\mathrm{F}$

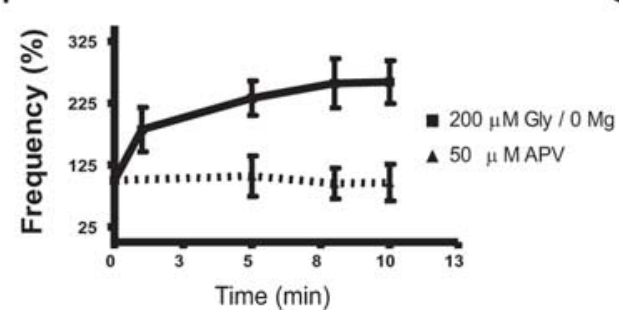

G

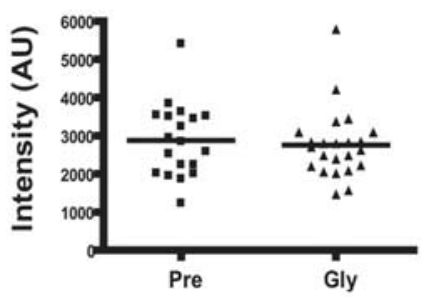

Figure 2. Resolution of discrete SEP-AMPAR exocytic events in dissociated pyramidal neurons. $\boldsymbol{A}$, Low-magnification view of SEP-AMPAR localization in a representative dissociated rat hippocampal neuron imaged using wide-field epifluorescence microscopy. The inset shows a portion of a dendrite at higher magnification. $\boldsymbol{B}$, Maximum projection image generated from a $60 \mathrm{~s}$ time series of sequential 100 ms TIR-FM images of a neuronal cell body, in which individual SEP-AMPAR insertions appear as bright spots. The inset shows sequential $100 \mathrm{~ms}$ TIR-FM images of the boxed area $(10 \times 10 \mu \mathrm{m})$. C, Example of a transient SEP-AMPAR insertion event observed in the dendrite; sequential $100 \mathrm{~ms}$ frames are shown, with the maximum intensity plot of the field plotted below. $\boldsymbol{D}$, Example of a persistent insertion event in a dendrite, with frames selected at the indicated time points after initial appearance of SEP-AMPAR fluorescence. $\boldsymbol{E}$, Representative maximum intensity plot before (left) and after (right) depletion of magnesium from the culture medium. $\boldsymbol{F}$, The squares and solid line indicate cumulative frequency analysis of SEP-AMPAR insertion events imaged during sequential $1 \mathrm{~min}$ intervals surrounding the indicated time points after $\mathrm{Gly} / 0 \mathrm{Mg}$ manipulation. Frequencies are normalized to $t=0$, the time interval immediately before magnesium depletion ( $n=6$ experiments representing 207 insertion events). The triangles and dotted line represent the same analysis conducted on cultures to which $50 \mu \mathrm{MAPV}$ was added 10 min before $t=0$ ( $n=4$ experiments representing 147 insertion events; error bars represent SEM; $\left.{ }^{*} p<0.05\right)$. G, Analysis of SEP-AMPAR insertion event peak fluorescence intensity in a representative field before (pre) and after Gly/0 Mg manipulation. 
A

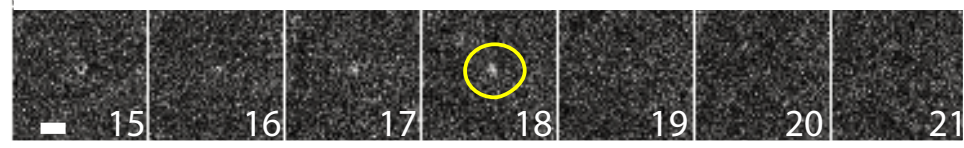

B
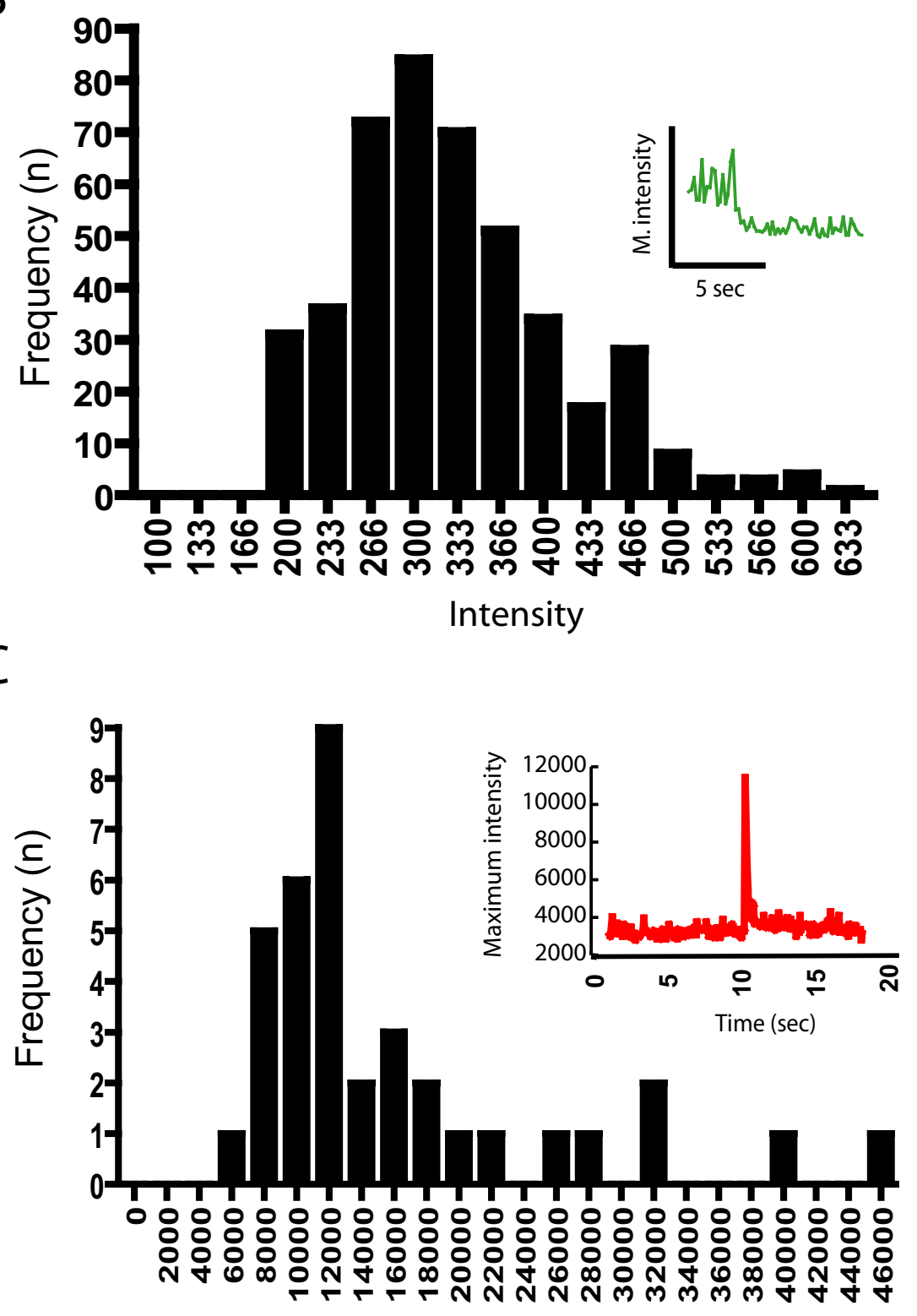

Intensity

Figure 3. Estimation of the number of SEP-AMPARs delivered to the plasma membrane by individual exocytic events. $\boldsymbol{A}$, Sequential $300 \mathrm{~ms}$ exposures from a representative field of surface-adsorbed EGFP imaged by TIR-FM, showing single-step photobleaching characteristic of individual molecules in the field. $\boldsymbol{B}$, Binned and background-subtracted intensity measurements of single EGFP molecules, compiled over multiple examples $(n=442)$ and normalized to $100 \mathrm{~ms}$ integration time (as described in Materials and Methods), yielded a mean single-molecule fluorescence intensity of 328 arbitrary units. The inset displays an example of an individual intensity trace showing single-step photobleaching. C, Binned and background-subtracted intensity measurements of SEP-AMPAR fluorescence intensities measured under the same conditions from 36 discrete exocytic events. The inset shows a representative intensity trace of a single event included in the distribution. The mean value of this distribution $\left(1.69 \times 10^{4}\right.$ arbitrary fluorescence units) yielded an estimate of $\sim 51$ labeled subunits inserted per exocytic event. number of SEP-AMPARs inserted from a single exocytic event was comparable with, or moderately in excess of, the number of receptors observed at steady state on spines.

Exocytic insertion of SEP-AMPARs occurs throughout the dendritic shaft but outside of synapses

Transient and persistent SEP-AMPAR insertion events were visualized throughout the length of the dendrite, from proximal to far distal portions, and often in regions of the dendrite projecting multiple spines (supplemental movie 4, available at www. jneurosci.org as supplemental material). Remarkably, however, all SEP-AMPAR insertion events observed in dendrites occurred outside of recognizable spines. This was true even in neurons previously photobleached, a manipulation used to reduce steady-state surface fluorescence and thereby increase detection sensitivity for exocytic events (see Materials and Methods). We were surprised by this observation because imaging studies were routinely conducted on mature cultures (DIV 15-20), in which synaptic spines are abundant and clearly visible in wide-field images (Craig et al., 1993; Eshhar et al., 1993; Rao et al., 1998). Nevertheless, it is conceivable that we could underestimate synaptic insertion by overlooking morphologically "stubby" spines (Ashby et al., 2006) or shaft synapses that are also present in these cultures (Bekkers and Stevens, 1989). To further investigate this issue, we examined the location of exocytic events relative to the postsynaptic density marker PSD-95, which is a reliable marker of both spine and shaft synapses (Kennedy, 1997; Kennedy and Ehlers, 2006). Because overexpression of PSD-95 can produce multiple effects on neuronal differentiation, dendritic morphology, and synaptic receptor localization (Bredt and Nicoll, 2003), we performed post hoc staining to detect endogenous PSD-95 in specimens after collecting live imaging data. This analysis confirmed that both transient and persistent SEPAMPAR exocytic events occurred in the dendritic shaft clearly outside of, although often in close proximity to, postsynaptic sites marked by endogenous PSD-95 (Fig. 4; supplemental movie 5, available at www.jneurosci.org as supplemental material).

Transient insertion events selectively promote lateral AMPAR delivery to nearby synapses

The localization of all observed exocytic insertion events outside of synapses raised the question of whether these events contribute AMPARs to the surface of synaptic 
spines. To address this, we used rapid imaging to examine the dissipation of SEPAMPARs from exocytic events occurring on the dendritic shaft. Transient events, apparently as a consequence of rapid lateral dispersion of inserted SEP-AMPARs, typically produced a visually apparent region of increased surface receptor fluorescence extending along the shaft from the site of exocytosis for a distance of several micrometers (typically 2-5 $\mu \mathrm{m}$ ) before dissipating to the basal level of surface concentration present in the surrounding plasma membrane (Fig. $5 A, B$ ). In some examples, this lateral spreading extended onto adjoining spines (an example is shown in Fig. 5C). Persistent insertion events, presumably because of their much slower dissipation, produced a less pronounced increase in locally spreading surface SEP-AMPAR fluorescence, despite having similar surface brightness on initial appearance (for example, this can be appreciated visually in supplemental movie 5, available at www.jneurosci.org as supplemental material). Accordingly, across multiple $(n=12)$ experiments in which hundreds of exocytic events were analyzed, persistent events were not found to visibly increase the surface fluorescence intensity of nearby spines.

Lateral spread of inserted SEPAMPARs from transient events markedly increased surface fluorescence on neighboring spines in some but not all examples, and apparent differences in surface delivery were noted even among individual spines located within the visible region of lateral spread. An example is shown in Figure $5 D$, in which a transient exocytic event (circled in red) produced a region of increased surface AMPAR fluorescence extending through the dendritic shaft (circled in orange) but visibly onto only one of two spines located nearby (circled in green and blue). Quantification of fluorescence intensity changes over time confirmed this lateral movement of inserted receptors selectively onto one of the nearby spines (Fig. $5 E$ ). On average, $\sim 25 \%$ of transient exocytic events visualized in the dendritic shaft produced a detectable increase in surface fluorescence on a spine located clearly within the "space constant" of lateral spread. The remainder of exocytic events analyzed dispersed through the shaft "nonproductively" (i.e., without producing a detectable increase in surface AMPAR fluorescence on any nearby spine) (Fig. 5F). Even among transient events promoting clearly visible lateral delivery of SEP-AMPARs to a spine, a significant fraction of the increased SEP-

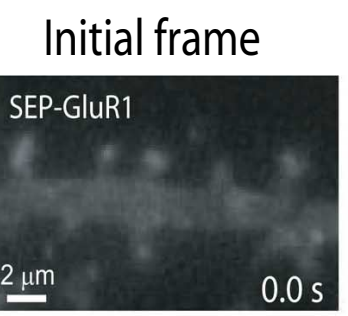

Transient event
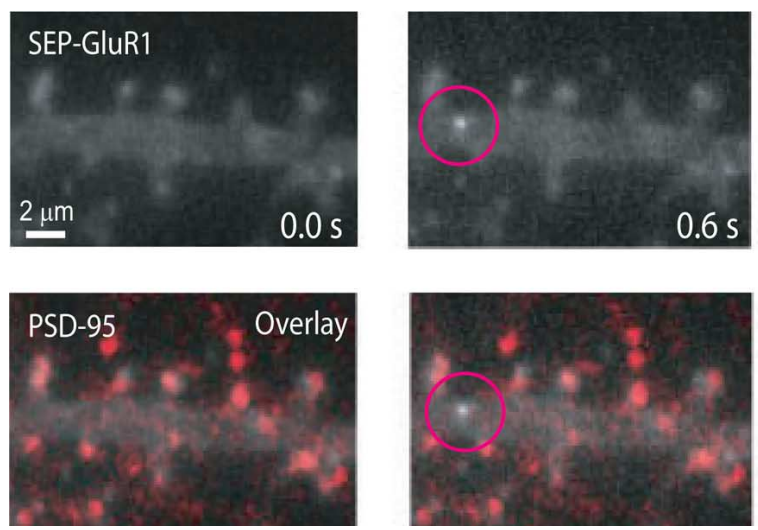

\section{Persistent event}
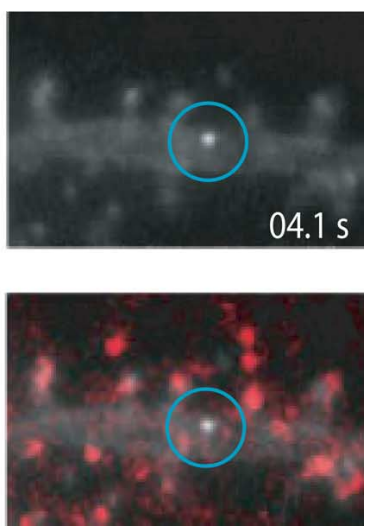

Figure 4. Localization of SEP-AMPAR exocytic events relative to the postsynaptic density. Wide-field image of SEP-AMPAR fluorescence (white) imaged in a region of a dendrite of a living pyramidal neuron in dissociated culture (top panels), and the same image overlaid with the localization of endogenous PSD-95 (displayed in red) determined by post hoc immunolabeling of neurons after chemical fixation (bottom panels). Selected $100 \mathrm{~ms}$ frames are displayed from a sequential image series, showing the initial image of the dendritic region (left), a transient exocytic event (middle), and a persistent exocytic event (right). The scale bar is in the bottom left, and the time stamp (in seconds) is in the bottom right. The entire kinetic series is included as supplemental movie 5 (available at www.jneurosci.org as supplemental material).
A
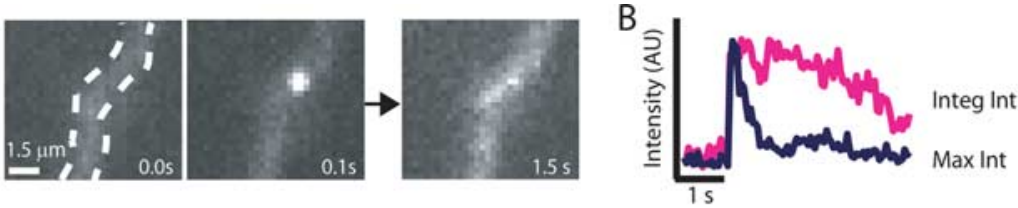

C
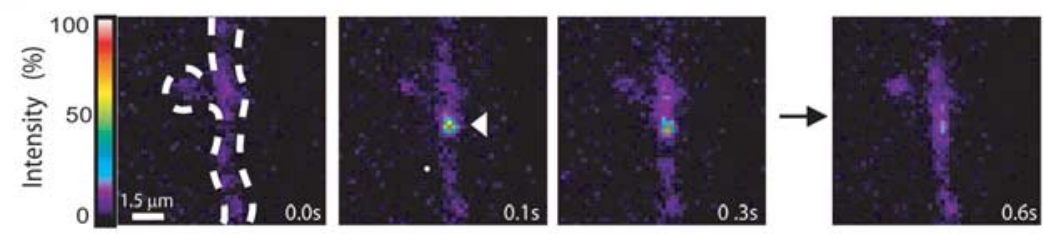

D

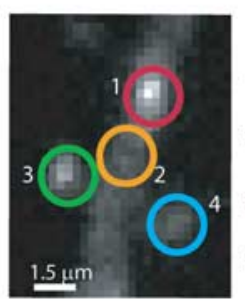

$\mathrm{E}$

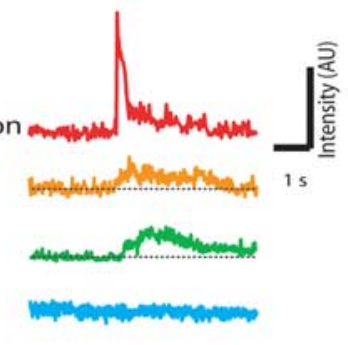

$\mathrm{F}$

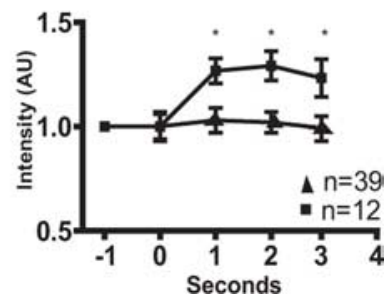

Figure 5. Lateral dispersion of SEP-AMPARs from individual exocytic events in the dendritic shaft. $A$, Locally increased surface fluorescence produced by dispersion of inserted SEP-AMPARs in the dendritic shaft. $\boldsymbol{B}$, Time course of maximal and integrated surface SEP-AMPAR fluorescence intensity from the dendritic field shown. Maximal intensity refers to the maximum pixel value in the field at the indicated time; integrated intensity refers to the fluorescence intensity summed over all pixels in the field shown. C, Sequential $100 \mathrm{~ms}$ image series showing dispersion of inserted SEP-AMPARs on the dendritic shaft and spread onto the surface of a nearby spine. Background-subtracted fluorescence intensities are displayed using the color scale indicated in the left panel. $\boldsymbol{D}$, Example of a single SEP-AMPAR insertion event (red circle) occurring in the local neighborhood of two opposing spines located near the site of insertion (green and blue circles). $\boldsymbol{E}$, Maximum intensity values over time of surface SEP-AMPAR fluorescence in the regions indicated in $\boldsymbol{C} . \boldsymbol{F}$, Quantification of SEP-AMPAR fluorescence imaged on randomly selected spines after an individual insertion event occurring $<5 \mu \mathrm{m}$ away on the dendritic shaft, where lateral spread of inserted SEP-AMPARs on the shaft domain immediately adjoining the spine was clearly evident. Data were collected from 51 separate insertion events occurring on different dendrites and in multiple neurons ( $n=5$ ), and binned according to whether (squares) or not (triangles) a detectable increase in surface SEP-AMPAR fluorescence was observed on the spine. Points represent mean change in maximum SEP-AMPAR intensity on the spine, averaged over the indicated $1 \mathrm{sintervals,} \mathrm{relative} \mathrm{to} \mathrm{that} \mathrm{measured} \mathrm{on} \mathrm{the} \mathrm{spine} 1 \mathrm{~s}$ before the initial appearance of the local SEP-AMPAR insertion event $\left(t=0\right.$ corresponds to the time at which the SEP-AMPAR insertion event was first detected; ${ }^{*} p<$ 0.05). Error bars represent SEM. 
AMPAR fluorescence appeared to exit the spine within several seconds after arrival (e.g., note that the increased SEP-AMPAR fluorescence evident in line 3 of Fig. $5 E$ returned nearly to baseline within several seconds). From the height of the residual "plateau" of SEP-AMPAR fluorescence intensity after this rapid decay phase, we estimated that $\leq 10 \%$ of SEP-AMPAR fluorescence delivered to a spine from a local exocytic event typically remained associated with the spine for a prolonged time period. Importantly, such rapid increases in SEP-AMPAR fluorescence on spines were observed only in the presence of an immediately preceding exocytic event located nearby. These results indicate that transient exocytic events are indeed capable of promoting local delivery of surface SEP-AMPARs to synaptic spines, but that synaptic delivery does not occur uniformly to all neighboring spines. Furthermore, the data suggest that local delivery by this mechanism occurs primarily to the mobile fraction of synaptic AMPARs (Triller and Choquet, 2005; Ashby et al., 2006; Sharma et al., 2006).

\section{Discussion}

To our knowledge, the present results are the first to directly visualize discrete membrane trafficking events mediating surface delivery of AMPARs. Our results unambiguously resolve both constitutive and regulated (NMDA receptor-dependent) components of exocytic insertion of GluR1-containing AMPARs in both hippocampal slice and dissociated culture preparations. Discrete insertion events were observed under basal conditions, and were substantially increased in frequency by two chemical stimuli that produce a net increase in surface AMPAR expression at steady state. Furthermore, our results reveal the existence of kinetically distinct exocytic modes mediating both constitutive and activitydependent surface delivery of AMPARs.

Previous studies have concluded variously that surface insertion occurs in the dendritic shaft, exclusively in the cell body, or directly into spines. They also differ on whether surface AMPAR insertion was proposed to occur in a regulated manner or constitutively (Passafaro et al., 2001; Ju et al., 2004; Sekine-Aizawa and Huganir, 2004; Adesnik et al., 2005; Gerges et al., 2006; Kopec et al., 2006). Such divergent conclusions are understandable given the previous inability to observe discrete AMPAR insertion events as they occur, and considering that the distribution of surface AMPARs can be rapidly modified by lateral movement (Triller and Choquet, 2005) or endocytosis (Malinow and Malenka, 2002). The present results, by directly resolving individual AMPAR insertion events in real time, show clearly that exocytic insertion of AMPARs occurs both in the cell body and throughout the full length of the dendritic shaft. Furthermore, in both compartments, our data identify constitutive and regulated components of exocytic insertion. The proportion of persistent exocytic events observed in dendrites was higher than that observed on the cell body. Nevertheless, estimates of absolute event frequency suggest that exocytic events occurring in both membrane domains make a significant contribution to net surface insertion of AMPARs. So far, we have not observed "hot spots" of repeated SEP-AMPAR insertion in either the cell body or in dendrites. Given that our rapid image series are limited for practical reasons to $1 \mathrm{~min}$ in length, however, we cannot rule out the existence of such regions of increased exocytic probability if repeated events occur $>1$ min apart.

We were surprised to note that, in multiple experiments in which hundreds of discrete exocytic events were visualized, exocytic insertion of AMPARs directly into spines was never observed. We cannot presently exclude the existence of such a direct exocytic route for synaptic AMPAR delivery, as suggested in an elegant recent study in which mutational inhibition of exocytic fusion produced an accumulation of intracellular AMPARs in spines at steady state (Gerges et al., 2006). Direct exocytic insertion to the spine could be missed by our methods if these events have relatively low surface fluorescence intensity. Nevertheless, considering the remarkably high peak surface fluorescence of the many exocytic events that our methods did resolve (and that all of these events occurred clearly outside of synaptic spines and postsynaptic sites marked by PSD-95), the present data argue strongly that a major pathway mediating both constitutive and regulated surface insertion of AMPARs is via exocytosis of AMPAR-containing vesicles occurring extrasynaptically in the dendritic shaft.

Given that many discrete exocytic events mediating surface AMPAR insertion were observed outside of synapses, how are receptors subsequently delivered to synaptic spines? One possibility is that surface delivery to spines occurs by lateral partitioning from a diffuse extrasynaptic receptor pool supported by longrange lateral diffusion (Groc et al., 2004; Adesnik et al., 2005; Cognet et al., 2006; Ehlers et al., 2007). Our results do not exclude this possibility, and, in fact, the occurrence of SEP-AMPAR exocytic events in the cell body is fully consistent with this hypothesis. Furthermore, we believe that the persistent exocytic events observed in the dendritic shaft, which dispersed slowly without producing a large increase in surrounding surface receptor fluorescence, likely deliver inserted AMPARs predominantly to the extrasynaptic pool. The present results also resolved a transient mode of exocytic insertion, however, which was clearly capable of producing increased surface receptor fluorescence on nearby spines. Thus, it is likely that transient exocytic events, in addition to contributing to the diffuse extrasynaptic receptor pool, can drive surface delivery of AMPARs preferentially to nearby spines. Accordingly, the existence of kinetically distinct exocytic modes suggests a simple principle by which exocytic events occurring in the dendritic shaft could permit local delivery of AMPARs to synapses (transient events) or effectively restrict delivery to a diffuse extrasynaptic pool (persistent events). The present study focused specifically on GluR1-containing AMPARs, whose rapid delivery to the plasma membrane is most firmly established to play a critical role in initiating synaptic potentiation under physiological conditions (Malinow and Malenka, 2002; Kopec et al., 2006). It will be interesting in future studies to determine whether transient and persistent exocytic events are mechanistically distinct, and to extend the analysis of discrete exocytic events to the surface delivery of other AMPARs, such as GluR2/3 heteromers thought to differ in exocytic trafficking properties (Passafaro et al., 2001; Triller and Choquet, 2005; Ashby et al., 2006). It will also be important to investigate whether local exocytic insertion driving lateral entry of AMPARs to synaptic spines functions separately from, or in concert with, synaptic stabilization of AMPARs mediated by spine-associated scaffold interactions (Schnell et al., 2002; Bats et al., 2007; Ehlers et al., 2007).

Dynamic AMPAR delivery to spines via lateral spread from transient insertion events, although less efficient when compared with direct insertion of receptors to the postsynaptic density, offers several potential physiological advantages. First, considering that pyramidal neurons can elaborate well over a thousand spines, regulated exocytosis in the shaft (in a local "neighborhood" of spines) could facilitate local delivery of AMPARs via intracellular trafficking machinery that may be shared by multiple synapses (Cooney et al., 2002). Second, because the duration of locally increased surface AMPAR concentration produced by 
transient insertion events is on a similar order as the mean estimated time for diffusive receptor entry through the spine neck (Holcman and Triller, 2006), local surface spreading as observed in the present study could optimally exploit the heterogeneous diffusion properties of neighboring spines (Ashby et al., 2006; Sharma et al., 2006) to promote synapse-specific delivery. Such kinetic control of surface delivery is likely to be particularly important for targeting the mobile fraction of AMPARs, which comprise a substantial fraction $(\sim 50 \%)$ of surface AMPARs present on spines at steady state (Triller and Choquet, 2005; Ashby et al., 2006; Sharma et al., 2006) and are not stably retained by scaffold protein interactions affecting the fixed pool of surface AMPARs (Groc et al., 2004; Gray et al., 2006; Bats et al., 2007; Ehlers et al., 2007; Ehrlich et al., 2007). Third, the incremental effect of individual exocytic events on increasing net surface AMPAR expression (Fig. 4E, F) suggests a simple "averaging" principle to reduce the stochastic noise in surface AMPAR insertion inherent to the regulation of individual exocytic events (Franks et al., 2003), potentially enhancing the reliability and precision of surface AMPAR delivery to individual synapses.

In conclusion, the present results provide unprecedented insight to the elemental basis of surface AMPAR delivery, and do so in a physiologically relevant population of excitatory neurons. Our data indicate that exocytic events occurring in both the cell body and dendritic shaft mediate constitutive and regulated surface insertion of GluR1-containing AMPARs, identify the frequency of discrete exocytic events as a major control point for NMDA receptor-dependent regulation of AMPAR delivery, and provide the first evidence for the existence of kinetically distinct exocytic modes that differ in propensity to drive local entry of AMPARs to postsynaptic sites. We propose that these events contribute specifically to the cell biology of activity-dependent synaptic plasticity, and may play a fundamental role in other processes of neural signaling and membrane remodeling as well.

\section{References}

Adesnik H, Nicoll RA, England PM (2005) Photoinactivation of native AMPA receptors reveals their real-time trafficking. Neuron 48:977-985.

Ashby MC, Maier SR, Nishimune A, Henley JM (2006) Lateral diffusion drives constitutive exchange of AMPA receptors at dendritic spines and is regulated by spine morphology. J Neurosci 26:7046-7055.

Bats C, Groc L, Choquet D (2007) The interaction between Stargazin and PSD-95 regulates AMPA receptor surface trafficking. Neuron 53:719-734.

Beattie EC, Carroll RC, Yu X, Morishita W, Yasuda H, von Zastrow M, Malenka RC (2000) Regulation of AMPA receptor endocytosis by a signaling mechanism shared with LTD. Nat Neurosci 3:1291-1300.

Beattie EC, Stellwagen D, Morishita W, Bresnahan JC, Ha BK, Von Zastrow M, Beattie MS, Malenka RC (2002) Control of synaptic strength by glial TNFalpha. Science 295:2282-2285.

Bekkers JM, Stevens CF (1989) NMDA and non-NMDA receptors are colocalized at individual excitatory synapses in cultured rat hippocampus. Nature 341:230-233.

Boehm J, Kang MG, Johnson RC, Esteban J, Huganir RL, Malinow R (2006) Synaptic incorporation of AMPA receptors during LTP is controlled by a PKC phosphorylation site on GluR1. Neuron 51:213-225.

Boulter J, Hollmann M, O'Shea-Greenfield A, Hartley M, Deneris E, Maron C, Heinemann S (1990) Molecular cloning and functional expression of glutamate receptor subunit genes. Science 249:1033-1037.

Bredt DS, Nicoll RA (2003) AMPA receptor trafficking at excitatory synapses. Neuron 40:361-379.

Carroll RC, Beattie EC, Xia H, Lüscher C, Altschuler Y, Nicoll RA, Malenka RC, von Zastrow M (1999) Dynamin-dependent endocytosis of ionotropic glutamate receptors. Proc Natl Acad Sci USA 96:14112-14117.

Cognet L, Groc L, Lounis B, Choquet D (2006) Multiple routes for gluta- mate receptor trafficking: surface diffusion and membrane traffic cooperate to bring receptors to synapses. Sci STKE 2006:pe13.

Cooney JR, Hurlburt JL, Selig DK, Harris KM, Fiala JC (2002) Endosomal compartments serve multiple hippocampal dendritic spines from a widespread rather than a local store of recycling membrane. J Neurosci 22:2215-2224.

Craig AM, Blackstone CD, Huganir RL, Banker G (1993) The distribution of glutamate receptors in cultured rat hippocampal neurons: postsynaptic clustering of AMPA-selective subunits. Neuron 10:1055-1068.

Ehlers MD, Heine M, Groc L, Lee M-C, Choquet D (2007) Diffusional trapping of GluR1 AMPA receptors by input-specific synaptic activity. Neuron 54:447-460.

Ehrlich I, Klein M, Rumpel S, Malinow R (2007) PSD-95 is required for activity-driven synapse stabilization. Proc Natl Acad Sci USA 104:4176-4181.

Eshhar N, Petralia RS, Winters CA, Niedzielski AS, Wenthold RJ (1993) The segregation and expression of glutamate receptor subunits in cultured hippocampal neurons. Neuroscience 57:943-964.

Franks KM, Stevens CF, Sejnowski TJ (2003) Independent sources of quantal variability at single glutamatergic synapses. J Neurosci 23:3186-3195.

Gerges NZ, Backos DS, Rupasinghe CN, Spaller MR, Esteban JA (2006) Dual role of the exocyst in AMPA receptor targeting and insertion into the postsynaptic membrane. EMBO J 25:1623-1634.

Gray NW, Weimer RM, Bureau I, Svoboda K (2006) Rapid redistribution of synaptic PSD-95 in the neocortex in vivo. PLoS Biol 4:e370.

Groc L, Heine M, Cognet L, Brickley K, Stephenson FA, Lounis B, Choquet D (2004) Differential activity-dependent regulation of the lateral mobilities of AMPA and NMDA receptors. Nat Neurosci 7:695-696.

Harris KM, Kater SB (1994) Dendritic spines: cellular specializations imparting both stability and flexibility to synaptic function. Annu Rev Neurosci 17:341-371.

Hayashi Y, Shi SH, Esteban JA, Piccini A, Poncer JC, Malinow R (2000) Driving AMPA receptors into synapses by LTP and CaMKII: requirement for GluR1 and PDZ domain interaction. Science 287:2262-2267.

Holcman D, Triller A (2006) Modeling synaptic dynamics driven by receptor lateral diffusion. Biophys J 91:2405-2415.

Ju W, Morishita W, Tsui J, Gaietta G, Deerinck TJ, Adams SR, Garner CC, Tsien RY, Ellisman MH, Malenka RC (2004) Activity-dependent regulation of dendritic synthesis and trafficking of AMPA receptors. Nat Neurosci 7:244-253.

Kennedy MB (1997) The postsynaptic density at glutamatergic synapses. Trends Neurosci 20:264-268.

Kennedy MJ, Ehlers MD (2006) Organelles and trafficking machinery for postsynaptic plasticity. Annu Rev Neurosci 29:325-362.

Kopec CD, Li B, Wei W, Boehm J, Malinow R (2006) Glutamate receptor exocytosis and spine enlargement during chemically induced long-term potentiation. J Neurosci 26:2000-2009.

Lin JW, Ju W, Foster K, Lee SH, Ahmadian G, Wyszynski M, Wang YT, Sheng M (2000) Distinct molecular mechanisms and divergent endocytotic pathways of AMPA receptor internalization. Nat Neurosci 3:1282-1290.

Lledo PM, Zhang X, Sudhof TC, Malenka RC, Nicoll RA (1998) Postsynaptic membrane fusion and long-term potentiation. Science 279:399-403.

Lu W, Man H, Ju W, Trimble WS, MacDonald JF, Wang YT (2001) Activation of synaptic NMDA receptors induces membrane insertion of new AMPA receptors and LTP in cultured hippocampal neurons. Neuron 29:243-254.

Luscher C, Xia H, Beattie EC, Carroll RC, von Zastrow M, Malenka RC, Nicoll RA (1999) Role of AMPA receptor cycling in synaptic transmission and plasticity. Neuron 24:649-658.

Malinow R, Malenka RC (2002) AMPA receptor trafficking and synaptic plasticity. Annu Rev Neurosci 25:103-126.

Miesenbock G, De Angelis DA, Rothman JE (1998) Visualizing secretion and synaptic transmission with $\mathrm{pH}$-sensitive green fluorescent proteins. Nature 394:192-195.

Moerner WE, Peterman EJ, Brasselet S, Kummer S, Dickson RM (1999) Optical methods for exploring dynamics of single copies of green fluorescent protein. Cytometry 36:232-238.

Otmakhov N, Khibnik L, Otmakhova N, Carpenter S, Riahi S, Asrican B, Lisman J (2004) Forskolin-induced LTP in the CA1 hippocampal region is NMDA receptor dependent. J Neurophysiol 91:1955-1962.

Park M, Penick EC, Edwards JG, Kauer JA, Ehlers MD (2004) Recycling endosomes supply AMPA receptors for LTP. Science 305:1972-1975. 
Passafaro M, Piech V, Sheng M (2001) Subunit-specific temporal and spatial patterns of AMPA receptor exocytosis in hippocampal neurons. Nat Neurosci 4:917-926.

Patterson GH, Knobel SM, Sharif WD, Kain SR, Piston DW (1997) Use of the green fluorescent protein and its mutants in quantitative fluorescence microscopy. Biophys J 73:2782-2790.

Rao A, Kim E, Sheng M, Craig AM (1998) Heterogeneity in the molecular composition of excitatory postsynaptic sites during development of hippocampal neurons in culture. J Neurosci 18:1217-1229.

Rumpel S, LeDoux J, Zador A, Malinow R (2005) Postsynaptic receptor trafficking underlying a form of associative learning. Science 308:83-88.

Sankaranarayanan S, De Angelis D, Rothman JE, Ryan TA (2000) The use of pHluorins for optical measurements of presynaptic activity. Biophys J 79:2199-2208.

Schnell E, Sizemore M, Karimzadegan S, Chen L, Bredt DS, Nicoll RA (2002) Direct interactions between PSD-95 and stargazin control synaptic AMPA receptor number. Proc Natl Acad Sci USA 99:13902-13907.

Sekine-Aizawa Y, Huganir RL (2004) Imaging of receptor trafficking by using alpha-bungarotoxin-binding-site-tagged receptors. Proc Natl Acad Sci USA 101:17114-17119.

Sharma K, Fong DK, Craig AM (2006) Postsynaptic protein mobility in dendritic spines: long-term regulation by synaptic NMDA receptor activation. Mol Cell Neurosci 31:702-712.

Sheng M, Lee SH (2001) AMPA receptor trafficking and the control of synaptic transmission. Cell 105:825-828.
Shepherd GM (1996) The dendritic spine: a multifunctional integrative unit. J Neurophysiol 75:2197-2210.

Shi G, Faúndez V, Roos J, Dell'Angelica EC, Kelly RB (1998) Neuroendocrine synaptic vesicles are formed in vitro by both clathrin-dependent and clathrin-independent pathways. J Cell Biol 143:947-955.

Shi SH, Hayashi Y, Petralia RS, Zaman SH, Wenthold RJ, Svoboda K, Malinow R (1999) Rapid spine delivery and redistribution of AMPA receptors after synaptic NMDA receptor activation. Science 284:1811-1816.

Stellwagen D, Malenka RC (2006) Synaptic scaling mediated by glial TNFalpha. Nature 440:1054-1059.

Stepanenko OV, Verkhusha VV, Kazakov VI, Shavlovsky MM, Kuznetsova IM, Uversky VN, Turoverov KK (2004) Comparative studies on the structure and stability of fluorescent proteins EGFP, zFP506, mRFP1, "dimer2," and DsRed1. Biochemistry 43:14913-14923.

Steyer JA, Almers W (2001) A real-time view of life within $100 \mathrm{~nm}$ of the plasma membrane. Nat Rev Mol Cell Biol 2:268-275.

Stoppini L, Buchs PA, Muller D (1991) A simple method for organotypic cultures of nervous tissue. J Neurosci Methods 37:173-182.

Triller A, Choquet D (2005) Surface trafficking of receptors between synaptic and extrasynaptic membranes: and yet they do move! Trends Neurosci 28:133-139.

Yudowski GA, Puthenveedu MA, von Zastrow M (2006) Distinct modes of regulated receptor insertion to the somatodendritic plasma membrane. Nat Neurosci 9:622-627. 